\title{
Introducing a Pre-Determined Feedback Pool for Evaluating Students' Online Discussion Participation
}

\author{
Dilani M. Perera-Diltz \\ University of Houston-Clear Lake \\ perera@uhcl.edu \\ R. J. Davis \\ University of Houston-Clear Lake \\ Sedef Uzuner Smith \\ University of Houston-Downtown \\ Carl Sheperis \\ University of Phoenix
}

\begin{abstract}
As self-regulation for successful online learning is a relatively new focus in online education, more attention is directed toward initiatives to help students become more cognizant of their learning efforts in online learning environments. Grounded in the idea that self-regulation is feedback dependent, we argue for the necessity of instructor feedback on student performance in online classes. The specific focus is instructor feedback on students' online discussion contributions. To assist instructors in managing their feedback practices in large online classes with numerous discussion entries, we present a feedback pool that includes a range of predetermined comments instructors can choose from while assessing their students' discussion participation.
\end{abstract}

Key words: instructor feedback, self-regulation, counselor education.

There is empirical consensus that discussions positively impact individual learning outcomes in online learning environments (e.g., Agee \& Uzuner Smith, 2011, Garrison \& Cleveland-Innes, 2005; Paus, Werner, \& Jucks, 2012; Vonderwell, 2003; Wu \& Hiltz, 2004; Zhu, 2006). Drawing on the work of scholars who established self-regulation as a pre-condition for successful online learning (e.g., Artino \& Stephens, 2009; Cho \& Shen, 2013; Paraskeva, Mysirlaki, \& Choustoulakis, 2009; Shea \& Bidjerano, 2010; Shea et al., 2012; Shen, Lee, \& Tsai, 2007; Sun \& Rueda, 2012; Wandler \& Imbriale, 2017), in this paper we contend that the benefit derived from discussions depends on the self-regulation abilities displayed in them. We define self-regulation in terms of "proactive processes that students use [...], such as setting goals, selecting and deploying strategies, and self-monitoring one's effectiveness” (Zimmerman, 2008, p. 166). Following this definition, we argue that discussions where students make effort to self-regulate their interactions in line with the discussion objectives have a high likelihood of generating conversations that offer meaningful learning opportunities for all parties involved. Conversely, discussions where participants do not make effort to self-regulate their interactions tend to do the opposite.

The argument that the degree to which students self-regulate their discussion participation has important implications for their own and their peers' learning begs the question: What triggers 
self-regulation? Although not specific to online discussions, in their description of motives to selfregulate learning, Zimmerman and Clearly (2009) made the argument that self-regulation is guided by feedback, and they identified three feedback sources that trigger self-regulation: "social (e.g., praise or guidance from a teacher, a peer, or a parent), environmental (e.g., task, microenvironment, or computer outcomes), and personal (e.g., awareness of covert, physiological, or behavioral outcomes)" (p. 247). We concur with Johnson and Johnson (1993) that "while the optimal situation may be to receive feedback concurrently [from all three sources], the most powerful source of feedback is from other people,” (p.136) and in the rest of this paper we turn our attention to a specific form of social feedback - feedback from the instructor.

Online teaching and learning literature recognizes feedback from the instructor as an important part of the learning process: "Feedback from the instructor is an important ingredient to the process because it lets students know when their thinking is on track and when it needs revised or redirected” (Lear, 2010, p. 1424). Here, we take this argument a step further and connect it to self-regulation and online discussions by stating that feedback from the instructor serves as a powerful means of directing students' attention toward the desired participatory behaviors in online discussions.

It is no surprise that not all students display the same participatory behaviors in online discussions. While some students mindfully engage with discussions, paying close attention to the details of their performance and being self-observant (aspects of self-regulation), others need to be nudged into doing so (Perera-Diltz \& Monaghan, 2014). We argue that individualized instructor feedback during discussions can benefit both groups of students: Feedback indicating deviation from desired expectations may help the latter group seek corrective actions; and feedback indicating successful performance may encourage the former group to continue their efforts to plan and reflectively monitor their discussion participation and behavior.

As Tan, Ullrich, Scheuer, Melis and Shen (2011) pointed out, some online classes have several hundred students. In such large classes, providing specific feedback that can bring about mindfulness in students and influence their self-regulated engagement with discussions can be a challenge for instructors. The contribution of this paper lies in its attempt to address this challenge by providing instructors with a feedback pool from which they can pick and choose appropriate comments and/or feedback applicable to various student behaviors when assessing discussion participation in large online classes.

The feedback pool we report here was developed through qualitative coding and analysis of discussion transcripts generated by students in fully online, graduate-level counselor education courses. The pool contains 47 comments subsumed under two feedback categories: growthoriented feedback and feedback for maintenance of desired behavior. In the sections below, we first describe the methods used to generate the feedback pool followed by a discussion on its significance, limitations along with future directions.

\section{Methods}

When we set out to develop the feedback pool, our interest was to access a large volume of student discussion data that could lead to a comprehensive analysis. Our context was an online counselor educator program at a public university in Southeast Texas. We began by identifying courses within this program that could yield rich student discussion data. Our search led us to two courses: Professionalism, Ethics, and Law; and Cross Cultural Counseling. Both courses were 5-weeks long and relied heavily on weekly discussions (each week had a discussion component). After obtaining 
Institutional Review Board approval, we accessed the student discussions generated in three sections of the Professionalism, Ethics, and Law course and three sections of the Cross Cultural Counseling course that were archived (offered in the 2012-2013 academic year). Each section (a total of six) consisted of approximately 20-25 students. The three sections of the Professionalism, Ethics, and Law course was taught by three different instructors but the learning outcomes, content, assignments, textbooks, and discussion activities were the same in all of them and they all followed the same syllabus. Their look and feel in Blackboard Learn (the learning management system that was used to deliver instruction) were also the same. This was the case for the three sections of the Cross Counseling course as well; they were taught by different instructors but had the same syllabus, same look and feel, same content, assignments, textbooks, and discussion activities.

In all sections, we focused on the discussions generated by students at the midpoint (Week 3) of the 5-week term. Our reasoning was that by this point in the term the students got accustomed to the structure of these courses and were able to sustain discussion without much prompting from the instructors.

The discussions in Week 3 yielded approximately 840 total pages of transcripts, roughly 130 to 140 pages per section. In the three sections of the Professionalism, Ethics, and Law course, the discussion for Week 3 was based on a scenario that required students to: (a) demonstrate decision-making using the ethical and legal guidelines they studied that week; and (b) describe their feelings during this decision making process. In the Cross Cultural Counseling course, the discussion for Week 3 required students to: (a) provide a synopsis of a movie that has a multicultural element; (b) identify positive and negative portrayals of different populations in the movie; and (c) discuss the degree to which the movie matched the information students studied that week about counseling American Indian and Asian American clients. (See Appendix 1 for full description of discussion prompts).

\section{Method of Analysis}

In the 840 total pages of discussion transcripts that we gathered from Week 3, we grouped the posts or messages from individual students together and labeled them as blocks. These blocks became our unit of analysis. We used inductive coding to analyze the blocks. Rather than analyzing the discussion transcript for each section in its entirety (i.e., 130 to 140 pages), we stopped coding when no new codes emerged due to saturation (Charmaz, 2006). We typically achieved saturation between pages 40 to 50 and these pages typically represented a minimum of 10 blocks (i.e., posts or messages by10 students). When we reached saturation in one section's transcript, we moved on to the next section's transcript. Using this method, we analyzed approximately 300 total pages of data.

We conducted the coding (using NVivo 10 qualitative analysis software) in two phases: Initial and focused coding (Charmaz, 2006). In initial coding, we read and reread the posts in each block to identify salient patterns, following Ryan and Bernard's (2003) useful list of things to look for, such as recurring regularities, similarities and differences across posts, and missing data (posting behaviors that students either intentionally or unintentionally avoided). Focused coding emerged from constant comparison of the initial codes with data. During focused coding, we made decisions to collapse or assign a new category for coded material. Below are two examples illustrating our coding process.

Example One. The first example comes from Sally (a pseudonym), a student from one of the Professionalism, Ethics, and Law sections. The block below contains her response to the 
scenario-based discussion prompt and the two other posts she made in response to other students. Post \#1: Date: January 30, 2013

Having "no experience in dealing with this type of client" I would aire on the side of caution and follow my supervisor's instructions to refer the client to the hospital. But prior to sending I would attempt to discuss with the client the situation in more detail and see if she would change her mind about involving the mother. Since the mother is there with her, it could breach her confidentiality by sending her while her mother is present. Hopefully, she would discuss in more detail her thoughts and I could assess whether or not involving her mother in the referral would be of harm.

There are many options: a. convince her to include her mother and gain her mother's support. After all, she did bring her mother to the counseling office. $b$. send her to the hospital per supervisor's instruction and disregard mother due to potential harm of patient to themselves. (not first option) c. allow her to go home but call her that evening and schedule a follow up appointment for next day (if mom is not permitted to be on board)

My feelings in the situation would be terribly conflicted. (due to the given of no experience) If the patient was indeed going to harm themselves, I would feel an ethical and moral responsiblity to get them immediate assistance. However, due to the HIPAA laws, and her mother being there....if the mom was not on the approved list then I just put my position at risk.

Primary ethical and legal concerns are: breaching confidentialy by involving mom without permission, liability concerns, HIPAA, losing my license and a client.

\section{Post \# 2: Date: February 1, 2013}

$\mathrm{Hi}$,

I am so sorry you went through that.

Like the passion you had to do the right thing and help your friend, I think it would be quite difficult to not assist the client who is considering suicide. It is a moral dilemna but true one that could cause us to get sued.

There is a HIPPA statement that allows certain family members information. The article did not state whether or not the mother was listed on the form as permitted to know. If she was on the statement, then the mom could know.

Post \# 3: Date: February 2, 2013

Oh that is good advice, thank you. I didn't state about documenting things. After the incident is concluded I would be sure to document the whole thing in my counselor notes too.

Good catch, thank you.

We identified elements in Sally's posts that could potentially be enhanced or improved 
with appropriate feedback. The first area that needed improvement in Sally's posting practices was her underdeveloped writing. In all three responses, Sally made general statements that did not provide any descriptive detail about the ideas she wants to convey. For example, in the first post, when talking about her options, she wrote she would "convince her [the client] to include her mother and gain her mother's support.” Sally did not give her readers any specific information or illustrations detailing what exactly she would do to convince the client. Similarly, Sally's last post was underdeveloped. Her statement, "I would be sure to document the whole thing in my counselor notes," did not give readers any indication of how or in what manner she will conduct this documentation. In her posts, Sally also appeared to make unsupported assertions without proper citation or evidence. She based all of her posts on personal opinion without making connections to the ideas/concepts that were presented in the reading assignment. At one point, she made a reference to a HIPPA statement, but rather than citing the statement and exploring its significance to the topic at hand, she moved ahead with personal opinion. Lastly, Sally's posts at times suffered from grammatical and mechanical errors, such as incomplete sentences (e.g., "Prior to sending I would attempt to ...”) incorrect spelling (e.g., "responsiblity” "dilemna” "confidentialy”) and punctuation errors that become distractions for the reader. Such problematic elements in Sally's posts can be targeted and discouraged through instructor feedback so she may consciously improve her discussion responses. Aside from these, there are aspects of Sally's posting practices that are laudable. One is that she showed engagement in the flow of the discussion by posting on different days. Students were required to distribute their posts evenly throughout the discussion period. She also met the minimum requirement for three weekly posts (minimum three substantive posts were required). These behaviors conducive to learning can be encouraged through instructor feedback.

Example Two. The second example comes from Joe (also a pseudonym), a student in one of the Cross Cultural Counseling sections. The block below contains his response to the discussion prompt (based on a movie) and the two other posts he made in response to other students.

Post \# 1: Date: December 5, 2012

Until I read the text for this week, I did not realize that the term "model minority" had been coined to refer to high achieving Asian students. For years, I was fascinated by my Asian students who seemed to be more focused, more responsible, more accountable, and more respectful than their peers of other backgrounds and cultures. I can remember writing a pretty lengthy paper on my Asian students and their culture while working on my certification through UTA. I wanted to know then what aspects of their culture lead them to set such high social, behavioral, and academic standards for themselves as a race of people. Baruth \& Manning (2012) state that the Asian culture emphasizes "diligence, harmony, taking responsibility, respect for authority, emphasis on education, respect for elders, and family loyalty." Although these attributes are important and generally instilled in Asian children, I have learned over the years that my thinking of Asian students as model minorities is stereotypical. Whereas I used to believe that every Asian student that I met would be high achieving and have a strong work ethic, I have come to find after 13 years of teaching that that simply is not true, and it is an "unrealistic expectation" and not fair to my students to make that assumption (Baruth \& Manning, 2012). I have encountered Asian students with varying academic ability levels, personalities, and behavioral patterns. Just as it is not fair to assume that 
all blacks are low performing and underachieving, it is not right to think that all Asian students are academically gifted.

In The Joy Luck Club, first generation Asian-American children struggle with their mothers over traditions, beliefs, and expectations. There are several ongoing themes throughout the movie: love, honor, respect, obedience, shame, forgiveness, perseverance, choices, and sacrifice. All of the women in the movie have gone through tremendous trials that have affected their lives in a multitude of ways, and they struggle to cope and make sense of their experiences. In the movie, many aspects from the text regarding the Asian culture are recognizable. For instance, Baruth \& Manning (2012) note that many Asian children become acculturated and adapt "to the norms and expectations of the dominant group." This is evident in the marriages of the daughters to white men despite the reluctance and concern on both sides. Acculturation is also noted in choosing Americanized names such as Waverly and Jennifer instead of traditional Chinese names. Rose hsu also states that she is "American" when speaking with Ted's mom, as if rejecting her cultural heritage to embrace her American roots. In addition, I noticed a sense of male dominance in the movie that the text references. Baruth \& Manning (2012) state that "children learn early on that the father is the head of the household in the traditional Asian American family." Furthermore, the text indicates that Asian people often do not seek counseling intervention when they are having mental health issues. It indicates that many Asians feel as if "talking about problems to outsiders is considered taboo," simply deny" that mental health issues exist, and do not receive help from family members (Baruth \& Manning, 2012). Throughout the course of the movie, communication between the family members is poor, and although the women truly have reasons to seek help, they do not make a decision to do so. Finally, it is evident that high expectations are placed on the children from a very early age. Whether it was a piano recital or chess tournament, the children were expected to practice, perform, and compete at high levels as to not shame the family. This coincides with the text's assertion that "Asian American parents who "have expectations of their children are unwilling to negotiate the terms" (Baruth \& Manning, 2012).

References

Baruth, L. G. \& Manning, M. L. T. (2012).Multicultural counseling and psychotherapy. Boston, MA: Pearson.

\section{Post \# 2: Date: December 7, 2012}

Hi Terri,

Although I have not seen The Help yet, based on the information that you provided about your husband's family, the story To Kill a Mockingbird comes to mind. In the novel set during The Great Depression, a widower, Atticus Finch, is responsible for caring for his two young children after his wife passes away. Since he is a lawyer and works long hours, the housekeeper, Calpurnia, takes on a maternal role for the children in the household. There is a tremendous amount of reciprocal love in the home; however, some people, namely Atticus' sister Aunt Alexandra, 
disapproves of the relationship. She feels that it is inappropriate for Calpurnia, a black woman, to be so familiar with the family, so she moves in to set things straight. Her intolerant, overbearing, racist mentality prompts her to destroy the close bond that her niece and nephew, Scout and Jem, have formed with the only mother-figure they have really known. For years "African-Americans have fought to overcome cultural dominance and discrimination" in situations such as the one described, and although so much has improved from efforts like the Civil Rights movement, racial prejudice still exists in our society (Baruth \& Manning, 2012). Counselors can help by advocating for people from all races and cultural groups in an effort to continue moving forward and help to increase tolerance, understanding, and equality for every citizen.

\section{References}

Baruth, L. G. \& Manning, M. L. T. (2012).Multicultural counseling and psychotherapy. Boston, MA: Pearson.

Post \# 3: Date: December 8, 2012

Hello Sue,

I enjoyed reading your post and learned a great deal from it. I watched A Time to Kill many, many years ago, but feel inclined to watch it again after reading your synopsis. It is often very difficult for me to read books or watch movies that deal with racism and discrimination. The abuse, injustice, and pain that is visited upon decent, law-abiding citizens simply because others are ignorant and intolerant is hard for me to comprehend. Two other movies came to my mind after reading your To Kill a Mockingbird and The Color Purple. To Kill a Mockingbird is one of our required 9th grade texts in my district, so every year, I get the opportunity to share insight with my students on the destructive nature of racism and discrimination. In this text, a hard working black man, Tom Robinson, is wrongly accused of raping a white woman in the South during the 1930's. And he, too, must rely on a white lawyer to vindicate him so that he can return home to his wife and children. Although things do not end well and he's subsequently found guilty and later killed in jail, his lawyer, an upstanding and honest southern gentleman, risks everything to clear Tom's name and teach his children lessons in integrity, sacrifice, and morality. The Color Purple, in my opinion, is one of the best novels of all times, and the movie is a great extension of the author's creation. Oprah Winfrey's character brings tears to my eyes every time I watch it. After standing up for herself against a white woman, she is jailed, separated from her children, and forced to become the woman's servant. Racism ultimately destroys her life as she rots away in jail and has her will and spirit broken senselessly by a society that believes in a racial supremacy and dominance. The black church also plays a huge role in the movie and has a significant impact on the resiliency of the character's. According to Baruth \& Manning (2012), counselors should understand that "religion and spirituality are deeply rooted in the African American culture" and provide black people with a sense of "connection, purpose, and empowerment."

\section{References}


Baruth, L. G. \& Manning, M. L. T. (2012). Multicultural counseling and psychotherapy. Boston, MA: Pearson.

Joe provided analytical and reflective responses in his first post. For example, in the first paragraph, he compared new knowledge (gained from the course readings) to past experiences (gained from personal experiences) and this comparison allowed him to approach the discussion content with considerable thought and reflection. In the second half of his first post, Joe explored and analyzed the movie in relation to the knowledge gained from the assigned readings. In his second and third posts, Joe took an interactive approach. He began with an affirmation of what others said and then developed the ideas with personal opinions and interpretations. Next, he took some of the ideas he read in the course readings and extrapolated them to what works in practice. His ability to move from personal exploration to idea extrapolation exhibits thoughtful and logical sequencing. Joe also showed engagement by posting on three separate days as the discussion unfolded rather than making all his contributions on the same day. Overall, Joe displayed posting behaviors that are desirable and should be reinforced with appropriate feedback.

\section{A Tentative Model of a Feedback Pool for Evaluating Students’ Online Discussion Participation}

Our qualitative analysis (which we illustrated with examples above) resulted in a tentative model of a feedback pool (See Table 1) that online instructors can use when evaluating students' discussion participation. The pool includes two types of feedback: growth-oriented feedback (i.e., feedback-indicating deviation from desired norms or expectations) and feedback for maintenance of desired behavior (i.e., feedback-indicating alignment with desired norms and expectations).

\section{Discussion}

\section{Significance}

In large online classes, providing students with specific and individualized feedback that promote their self-regulated engagement with discussions can be a challenge for instructors. In this paper, we propose a feedback pool as a potential solution to this problem. We believe that the availability of a multitude of predesigned comments to choose and personalize from can transform online instructors' feedback giving practices, including the content of the feedback as well as its timing, efficiency, and management. More importantly, receiving feedback not just a grade will potentially influence students' intentional self-regulation, leading them to monitor their discussion performance and strategically plan for improvements.

Online instructors interested in using this feedback pool can utilize it in the way that best serves their needs. They can personalize the feedback comments or change them as appropriate for individual students. When using the feedback pool, we recommend that instructors gauge how much feedback to provide and which categories to focus on. For both instructors and students, selective and focused feedback (i.e., feedback focusing on two or three categories rather than all or most categories all at once) will be more manageable.

\section{Limitations}


A limitation of the feedback pool presented here is that it was developed with discussion data obtained from counselor education courses. However, since the comments presented in the pool address various forms of discussion participation behaviors that are not subject-specific, they can be used in online courses in disciplines other than counseling. Perhaps one can argue that the comment pool is more appropriate for graduate level courses, especially those in social sciences. One way to see whether this is in fact the case is to ask this question: Is the comment pool going to be used for discussions requiring students to show understanding and synthesis of ideas and to collectively build on each other's ideas? If the answer is yes, then the pool is very likely to be applicable.

Another limitation is about the caveats to consider when using feedback from a pool of pre-determined comments. First and most important, when students repeatedly receive the same feedback (in which the same words are used) or receive the same feedback as others, they may start perceiving such feedback as canned, rather than authentic. As such, while pre-constructed feedback pools are helpful for instructors with large class sizes, they must be carefully utilized so as not to give students the impression that their performance is not monitored and evaluated with scrutiny. Secondly, no pre-determined feedback pool can be exhaustive. There may be instances where the comments in the pool we provide will not suffice. Thirdly, when using comments from a feedback pool, instructors need to make sure that the comment they select from the pool actually reflects the appropriate or intended message that needs to be communicated to a student (e.g., instructor indicates a change should be made in a student's posting behavior when in actuality that change is not needed).

\section{Future Directions}

In a recent Faculty Focus article on tips for effective and efficient grading, Smith and Palenque (2015) suggested, "Keep a bank of comments about frequent errors students make and organize them in groups for easy access.” The feedback pool presented in this paper is an actual realization and implementation of this recommendation. To improve on the work that has begun with the generation of this feedback pool, there is a need to develop a feedback plug-in application for any learning management system to allow instructors to drag and drop appropriate comments from the pool and insert them into their evaluation of individual students' performance on discussion. 
Table 1. Tentative Model of a Feedback Pool for Evaluating Students' Online Discussion Participation

\begin{tabular}{|c|c|c|c|}
\hline Category & Posting behavior & Growth-oriented feedback & $\begin{array}{l}\text { Feedback for maintenance of desired } \\
\text { behavior }\end{array}$ \\
\hline $\begin{array}{l}\text { Cognitive } \\
\text { processes }\end{array}$ & $\begin{array}{l}\text { Textual } \\
\text { engagement }\end{array}$ & $\begin{array}{l}\text { The majority of your post is based } \\
\text { on personal opinion/experience } \\
\text { without any connection to the } \\
\text { assigned text(s). Next time, start } \\
\text { with what you read (i.e., theory, } \\
\text { research, methods, etc.) and expand } \\
\text { upon that information to } \\
\text { incorporate your personal } \\
\text { experiences/opinions/interpretation. }\end{array}$ & $\begin{array}{l}\text { Your post demonstrates clear } \\
\text { connections to the assigned text(s) } \\
\text { (and/or relevant outside resources) } \\
\text { while maintaining a personal voice. } \\
\text { Writing in this manner (weaving } \\
\text { analysis/synthesis and personal } \\
\text { interpretations/evaluations into a } \\
\text { coherent whole) enhances your } \\
\text { contribution to the discussions. }\end{array}$ \\
\hline
\end{tabular}

Reflection $\quad$ Your post would be enhanced by including your reflection. Rather than listing personal experience, explain how you will build on your prior experiences to respond to new and challenging situations. Or rather than listing information, explain what you have learned and what else you need to inquire about.

Connecting experience to learning
Use personal experience or anecdotes judiciously. If appropriate (and only for illustration of something), relate the topic to your personal experience and be sure to make the link between the experience and the course material.
By explaining what changes happened in your learning, what you need to improve on or inquire about, you are demonstrating reflection in your posts, which adds value to your contribution(s).

Your judicious use of personal experience (or anecdotes) enriched your writing and allowed you to make meaningful connections between the course material and the real world. 


\section{Making}

assertions

Relevance

Analyzing

Illustrating

Probing
You have stated information as factual or made sweeping generalizations without proper support or evidence. Your writing would be enhanced if you supplied reasons for your opinions or explained how you came to your ideas(reached your conclusions).

Your writing strays off topic into areas that do not relate to the topic of discussion. Please only include material that relates to the topic at hand.

Your writing would be strengthened if you engaged in some analysis by identifying or examining connections, patterns, differences or similarities between the text and your experiences OR between/among the ideas presented.

Your posts would be strengthened if you utilized examples that illustrate the points you wish to make rather than simply listing information or generalizations about a topic.

Your posts would be strengthened with probing questions that move the discussion forward.
In your post, you have supported your own points/opinions with relevant facts and evidence. This allows you to justify your reasoning and enhances the accuracy of what you have written.

You write honing in on the topic. This helps the reader understand your insight related to the topic.

By making connections between the text and your experiences and/or extending the ideas by identifying the similarities and differences in them, you are displaying high-level thinking which elevates the discourse.

Your exploration of the course content through the use of examples in your post enhances your writing and facilitates understanding.

The thought-provoking questions you asked in your post(s) enhanced the discussion and engaged your peers. 
Engagement

\section{Responsive}

interaction

Posting Practices

Focus

(In)complete

response

(Under)developed response
Your discussion contributions would be strengthened if you engaged more actively with your peers by building on or inviting their comments.

Duplicate Posts: Your posts are identical with each other.

Meaningful contribution to the discussions requires new and additional insights.

Singular Focus: The content of your posts are largely a re-statement of prior posts. Please avoid repetition of content. For example, you may choose to add new, different or contrasting ideas and information which has the potential to extend the discussion.

Your post does not achieve the purpose of the discussion. When a prompt or guide is provided, you are expected to address all of its components.

Your posts include superficial comments (such as 'I agree/disagree" or "I like" statements) which do not reflect indepth engagement.
You are actively engaged with your peers by building on or inviting their comments.

Each of your posts provides meaningful contributions to the discussion.

Each of your posts bring new ideas or a novel way of looking at previously stated information.

Your post achieves the purpose of the discussion by addressing all components of the prompt.

You post(s) includes properly developed ideas with details and explanations. 
Some ways to achieve sufficient depth in your writing include developing ideas by adding more detail, elaborating on the meaning you wish to convey, or exploring alternative perspectives.

Full participation

No participation

Partial

participation

(Un)even

participation

$\begin{array}{ll}\text { Written } & \text { Punctuation \& } \\ \text { expression } & \text { Spelling }\end{array}$

You have not participated in the discussion. Please review the course expectations with regard to the number of required posts and contact me if you have questions.

You have only partially participated course expectations with regard to the number of required posts.

Your posts are not evenly distributed throughout the discussion period. All of your posts occur on the same day or on the weekend. Please review the course expectations with regard to the frequency of required posts.

Your post includes numerous errors in spelling, punctuation, and/or capitalization that make the reader struggle through the writing. In addition, these mistakes have the in the discussion. Please review the
You are fully engaged in the discussion with evenly distributed contributions.

Your posts are well spaced throughout the week indicating active engagement in discussion

Your post demonstrates strong control of standard writing conventions, such as punctuation, spelling, and capitalization. 


\section{Grammar and usage}

Word choice

Disciplinespecific terminology potential to cause the reader to question the effort and care you put into your writing. Please use the writing tools available to you, such as spell check, to improve the quality of your writing and edit/proofread your writing before posting it.

Your writing displays repeated grammatical errors that impede meaning or distract the reader. Please proofread your writing or utilize a grammar checking software.

Your writing suffers from poor or incorrect word choice. Academic writing requires a certain level of sophistication wherein slang is avoided and high-level vocabulary is appropriately utilized.

In order to elevate the discourse to the appropriate academic level, you should pay careful attention to word selection in your posts.

When appropriate, use correct and relevant discipline-specific terminology to manage the complexity of the topic.
Your post demonstrates strong control of grammar and usage.

Through careful attention to word selection, your writing elevates the level of the discourse to the appropriate academic level.

Your post(s) demonstrates correct and relevant discipline-specific terminology. 
(Over)use of first person You are overusing first-person language-singular language (I) in your posts. Using the first person is acceptable in some forms of academic writing. However, overuse of first person language detracts from the quality or scholarly nature of the writing. Please avoid multiple or continuous use of sentences that begin with (I).

Overuse of first person languageplural

\section{Organization}

Use of In-text citations

source material

You are using first-person plural (we) in your posts. Using the first person plural (we) detracts from the clarity of your writing. In other words, it is often unclear who "we" is referring to in the writing.

Your post demonstrates a weakness in organization. Please pay attention to the overall coherence of your ideas. Avoid writings that lack focus or contain digressions or repetitions.

In-text citation(s) included in your post is too general in nature. A clear connection between the points/ideas in your post and your citation(s) is not apparent. When integrating sources into your own sentences and paragraphs, please remember to introduce the source to your readers first. One way to introduce the
Your careful use of first-person language in your posts is appropriate
Your post exhibits thoughtful and logical progression of ideas.
You are appropriately integrating sources (through quoting and paraphrasing) into your own sentences and paragraphs. It is evident that you understand the strategy of introducing sources to clarify where the ideas came from and why they are used. 
(Over)reliance

on direct quotes

Format

Formatting 1

Formatting 2 source or to let the reader know that you are transitioning from your own thoughts to the thoughts, writing, or work of others is by using signal phrases such as "According to (author name) ..." or "(Author name) states that ..."

In your post, you are relying on lengthy, direct quotes as a substitute for personal meaning making and interpretation of the source material. Direct quotes are appropriate and often necessary to support specific ideas. However, your voice as a writer should be clear and evident alongside your citations or reference to the work of others.

The references and in-text citations you included in your post deviate from the APA format. Please familiarize yourself with current APA guidelines. I am happy to discuss specific details with you should you have any questions.

The references and in-text citations included in your post(s) are wrong (inaccurate author names, year, page numbers, etc.,) or incomplete. Please make sure to cite material
Your restrained use of direct quotes enhances the communication of your position

Thank you for following the APA format correctly and consistently in your writing. 
correctly in the body of your post(s)

and in your reference list.

Formatting 3

The citations within the body of your post(s) generally occur outside of sentence punctuation. Please make sure to place citations at the end of a sentence before a period. When you place a citation within a sentence, make sure that it appears before internal punctuation such as a comma or semicolon.

Formatting 4

Please remember that every source in the reference list must be referred to at least once within the body of your post. If no citation appears in your writing, a resource cannot be included in the references. 


\section{Acknowledgements}

This research was grant funded by Academic Partnerships and some parts of this paper are published as a grant report on Academic Partnership's website at:

http://facultyecommons.com/spotlight-lamar-university-ap-faculty-research-grant-recipients/

\section{Appendix}

\section{Appendix 1. Description of Discussion Prompts.}

Week 3 Discussion Prompt in Professionalism, Ethics, and Law Course

For this week's discussion forum, respond to the following case study and discussion questions:

Case Study: You are a counselor at a community based mental health clinic. You mainly see children, adolescents, and their families. One day you are filling in for the intake counselor. Marcie is a new client, a 22-year-old female who works as a receptionist in a doctor's office. She has just revealed to you during the intake session that she has recently lost interest in most activities, has been sleeping a great deal yet feels tired all the time, and sometimes wishes she could cease to exist. She mentioned feeling as though she were "on an emotional roller coaster" during the past year, throughout her on-again, off-again relationship with a 35-year-old married man. The last breakup with him seemed really final, and Marcie has felt herself sinking deeper and deeper ever since. When probed further about suicidal ideations, Marcie admitted that she has considered killing herself although she is uncertain whether or not she would actually do it. She said that she is currently in possession of a gun her friend allowed her to keep in her home following a rash of burglaries in the neighborhood, but she doesn't know whether she would actually use it. You have consulted with your supervisor, who has agreed that Marcie should be referred immediately for a psychiatric evaluation and has instructed you to arrange for Marcie to go directly from your office to a nearby hospital. Marcie told you that her mother accompanied her to this appointment and is in the waiting room, but she has emphatically stated that she does not want her mother to know what is going on with her. You have had no experience in dealing with this type of client. How should this delicate situation be handled?

\section{Discussion Questions:}

What should you do?

What options do you have?

How did you feel as you thought about how you would handle this situation?

What was your primary ethical and legal concerns related to this case?

Initial Post: You should make sure your post thoroughly addresses all parts of the assignment. Your comments should be clearly and concisely stated, demonstrating that the content was appropriately reviewed and synthesized. You should be sure to cite your points using the text, learning resources, and other scholarly literature to support your discussion. You must use appropriate APA citations and references. 
Peer Responses: Multiple posts are made offering substantial, well written contributions, opinions, observations, questions, experiences, critiques, suggestions, etc. Citations must be included. Comment on ethical codes or legal precedents that might relate to your peers' personal experiences. Peer responses must occur on at least two separate days.

Remember: In order to meet minimum requirements, you must respond with your initial post by day 4 (Thursday) and respond to at least 2 peers with substantive responses and support these responses with references. Posts should be evenly distributed throughout the discussion period, reflecting participation from start to finish. All posts should be appropriate, using proper language and mechanics.

\section{Week 3 Discussion Prompt in Cross Cultural Counseling Course}

For this week's discussion forum, respond to the following questions:

This week you read about counseling American Indian and Asian American clients. You were exposed to the various issues that impact the counseling process for different populations. As part of preparing for this week's discussion, read your course materials and then watch one of the movies listed in your course resources for this week (file name is Multicultural Movies_). You can access most of the movies via Amazon Instant Video, Netflix, or any other video source. You can also purchase the movies via Amazon and other vendors.

For this week's discussion forum, respond to the following prompts/questions:

1. Provide a synopsis of the film you watched and the top three multicultural issues that stood out for you.

2. Provide specific examples in which the film influences (positively or negatively) perceptions of multicultural issues. Be sure to identify any potential for the movie to promote bias.

3. Discuss the degree to which the film you watched matches the information you have learned in this course

Use examples to support your ideas. Be sure to cite your points using the learning resources. You may also use other scholarly literature to support your discussion. Citations and references must be in APA format. See the grading rubric for more information.

Peer Response: Respond to a MINIMUM of 2 peers with substantive responses and support these responses with references. Peer responses must occur on two separate days. See the grading rubric for more information.

Before responding to your colleagues, watch one of the films on Asian Americans or American Indians. Compare and Contrast the attitudes, beliefs, understandings, and acculturative experiences inherent in these films to those in the main post. Also contribute to the discussion by building on examples or providing your own in relation to their main discussion post.

Posting for the discussion board are due by Day 4, and responses are due by Day 6. 


\section{References}

Agee, J. and Uzuner Smith, S. (2011). Online discussions in a doctoral research methods course: Like a text by many authors. Studies in Continuing Education, 33(3), 301-319.

Artino, A. R. and Stephens, J. M. (2009). Academic motivation and self-regulation: A comparative analysis of undergraduate and graduate students learning online. The Internet \& Higher Education, 12, 146-151.

Charmaz, K. (2006). Constructing grounded theory: A practical guide through qualitative analysis. Thousand Oaks, CA: Sage.

Cho, M-H. and Shen, D. (2013). Self-regulation in online learning. Distance Education, 34(3), 290-301.

Garrison, D. R. and Cleveland-Innes, M. (2005). Facilitating cognitive presence in online learning: Interaction is not enough. The American Journal of Distance Education, 19(3), 133-148.

Johnson, D. W. and Johnson, R. T. (1993). Cooperative learning and feedback in technology-based instruction. In J. V. Dempsey and G. C. Sales (Eds.), Interactive instruction and feedback. (pp. 133-158). Englewood, NJ: Educational Technology.

Lear, J. (2010). Instructor presence in online distance classes. In Web-based education: Concepts, methodologies, tools, and applications. (pp. 1422-1427). Hershey, PA: Information Science Reference.

Paraskeva, F., Mysirlaki, S. and Choustoulakis, E. (2009). Designing collaborative learning environments using educational scenarios based on SR. International Journal of Advanced Corporate Learning, 2(1), 42-49.

Paus, E., Werner, C. S. and Jucks, R. (2012). Learning through online peer discourse: Structural equation modeling points to the role of discourse activities in individual understanding. Computers \& Education, 58, 1127- 1137.

Perera-Diltz, D. M. and Monaghan, C. H. (2014). A dialogue on strategies for effective online counselor education instruction. In G. R. Walz and J. C. Bleuer, (Eds.), VISTAS Online 2014 (article 54). Alexandria, VA: Counseling Outfitters. Retrieved June 9, 2017, from http://www.counseling.org/knowledge-center/vistas

Ryan, G. W. and Bernard, H. R. (2003). Techniques to identify themes. Field Methods, 15, 85109.

Shea, P. and Bijerano, T. (2010). Learning presence: Towards a theory of self-efficacy, selfregulation, and the development of communities of inquiry in online and blended learning environments. Computers \& Education, 55, 1721-1731.

Shea, P., Hayes, S., Uzuner, S., Vickers, J., Bijerano, T., Pickett, A. and Jian, S. (2012). Learning 
presence: Additional research on a new conceptual element within the community of inquiry (CoI) framework. The Internet and Higher Education, 15(2), 89-95.

Shen, P. D., Lee, T-H. and Tsai, C-W. (2007). Applying Web-enabled problem-based learning and self-regulated learning to enhance computing skills of Taiwan's vocational students: A quasiexperimental study of a short-term module. Electronic Journal of e-Learning 5(2), 147-56.

Smith, V. and Palenque, S. M. (2015, February 2). Ten tips for more efficient and effective grading. Faculty Focus. Retrieved June 9, 2017, from https://www.facultyfocus.com/articles/educational-assessment/ten-tips-efficient-effectivgrading/

Sun, J. C-Y. and Rueda, R. (2012). Situational interest, computer self-efficacy and self-regulation: Their impact on student engagement in distance education. British Journal of Educational Technology, 43(2), 191-204.

Vonderwell, S. (2003). An examination of asynchronous communication experiences and perspectives of students in an online course: A case study. The Internet and Higher Education 6, 77-90.

Wandler, J. and Imbriale, W. (2017). Promoting undergraduate student self-regulation in online learning environments. Online Learning, 21(2).

Wu, D. and Hiltz, S. R. (2004). Predicting learning from asynchronous online discussions. Journal of Asynchronous Learning Networks, 8(2), 139-152.

Zhu, E. (2006). Interaction and cognitive engagement: An analysis of four asynchronous online discussions. Instructional Science, 34, 451-480.

Zimmerman, B. J. (2008). Investigating self-regulation and motivation: Historical background, methodological developments, and future prospects. American Educational Research Journal, 45(1), 166-183.

Zimmerman, B. J. and Clearly, T. J. (2009). Motives to self-regulate learning: A social account. In K. R. Wentzel and A. Wigfield (Eds.), Handbook of motivation at school (pp. 247-264). New York, NY: Routledge. 